\title{
Research on Series Arc Fault Characteristics Based on BSS and Harmonic Analysis
}

\author{
Zheng Wang \\ Quanzhou Yixing Electric Power Co., Ltd \\ Quanzhou, China \\ qzwangz@163.com
}

Kai Yang *

College of Mechanical Engineering and Automation

Huaqiao University

Xiamen, China

yklunw@sina.com

* Corresponding Author

\author{
Fengming Huang \\ Quanzhou Yixing Electric Power Co., Ltd \\ Quanzhou, China \\ qzwangz@163.com
}

\author{
Qiuhong Chen \\ Quanzhou Yixing Electric Power Co., Ltd \\ Quanzhou, China \\ qzwangz@163.com
}

\author{
Shouhong Chen \\ College of Mechanical Engineering and Automation \\ Huaqiao University \\ Xiamen, China \\ shouhong55@163.com
}

\begin{abstract}
Arc fault is one of the most important reasons of electrical fires. The common characteristics of series arc faults are always submerged in load currents which are acquired as mixed signals. It is difficult to extract useful arc fault characteristics through conventional approaches. Hence, novel approaches based on blind source separation and harmonic analysis are developed for characteristic research of series arc faults. At first, a low-voltage arc fault experimental platform was set up to generate arc faults of different loads, and a large number of load currents were acquired to analyze fault characteristics. When series arc faults occurred in circuits, currents would distort a little. Then, in order to obtain current minor changes, blind source separation (BSS) was used to separate distortions from observed mixed signals. Finally, common characteristics of series arc faults were found. The amplitudes of odd harmonic components in arc fault currents change rapidly. The total odd harmonic ratio fluctuates tempestuously with inconstancy but increases a lot. These harmonic characteristics provide very important bases for arc fault detection.
\end{abstract}

Keywords: Series Arc Fault; Blind Source Separation; Current Distortion; Harmonic Analysis; Harmonic Ratio

\section{INTRODUCTION}

In the light of fire statistical data from official fire services, short circuit faults, over current faults, leakage faults and arc faults result in over $90 \%$ of electrical fires in people's daily lives [1,2]. In the present, over current protectors are usually used to detect over current faults in electrical circuits, and leakage protectors are always used to diagnose leakage faults in electrical circuits. Both of them cannot be used to detect arc faults which are always classified three major categories: parallel arc fault, series arc fault and ground arc fault. The characteristics of parallel arc faults are similar to over current faults, and the characteristics of ground arc faults are similar to earth faults. Thus, ground arc fault and parallel arc fault are not difficult to be detected [3]. Nevertheless, series arc fault currents are usually easily submerged by loads' currents and strong background interference. Thus, it is difficult to extract series arc fault features from different kinds of load mixed signals, and it is also quite difficult for us to diagnose series arc faults accurately as there is not reliable series arc fault features. In virtue of cross talk, weakness and randomness of arc fault signals, a lot of conventional approaches have all sorts of limitations. When arc faults occur in low-voltage circuits, there are some abnormal characteristics on arc radiation signals, arc light, arc temperature and arc voltage appeared on fixed sites, they can be used to identify arc faults [4-6]. However, the generation site, time and intensity of series arc faults have some randomness. Therefore, there are some difficulties to put these approaches into practice. What's more, some advanced approaches on arc mathematical model establishments always just stay in the simulation state [7-9]. Therefore, it is significant to extract series arc fault characteristics from different kinds of load mixed signals accurately. In this paper, during the whole research process of series arc fault characteristics, in order to heighten the accuracy of fault characteristic extraction under the surroundings of many types of interference and noise, this paper acquires a large quantity of series arc fault mixed signals to study the 
common characteristics of different loads. At first, independent current components are separated from observed current mixed signals through blind source separation (BSS). Then, harmonic analysis method is used to extract series arc fault characteristics from different load signals. Finally, general characteristics of series arc faults are found. The detailed series arc fault characteristic research method is developed in this paper.

\section{SET UP EXPERIMENTAL PLATFORM}

To research series arc fault characteristics, a lowvoltage arc fault experimental platform is set up as shown in Fig. 1, which is in accordance with the lowvoltage arc fault detection standards. And criterions contain UL1699, GB/T 31143-2014 and IEC 62606: 2013 are used as referential specifications to set up that arc fault experimental platform.

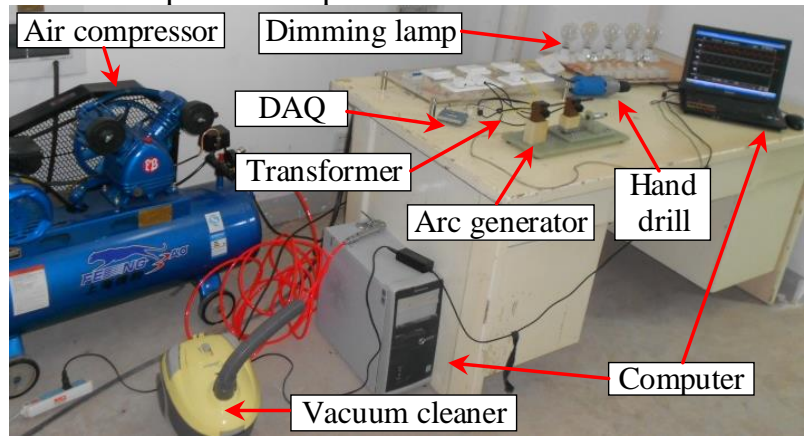

Figure 1. Low-voltage arc fault experimental platform.
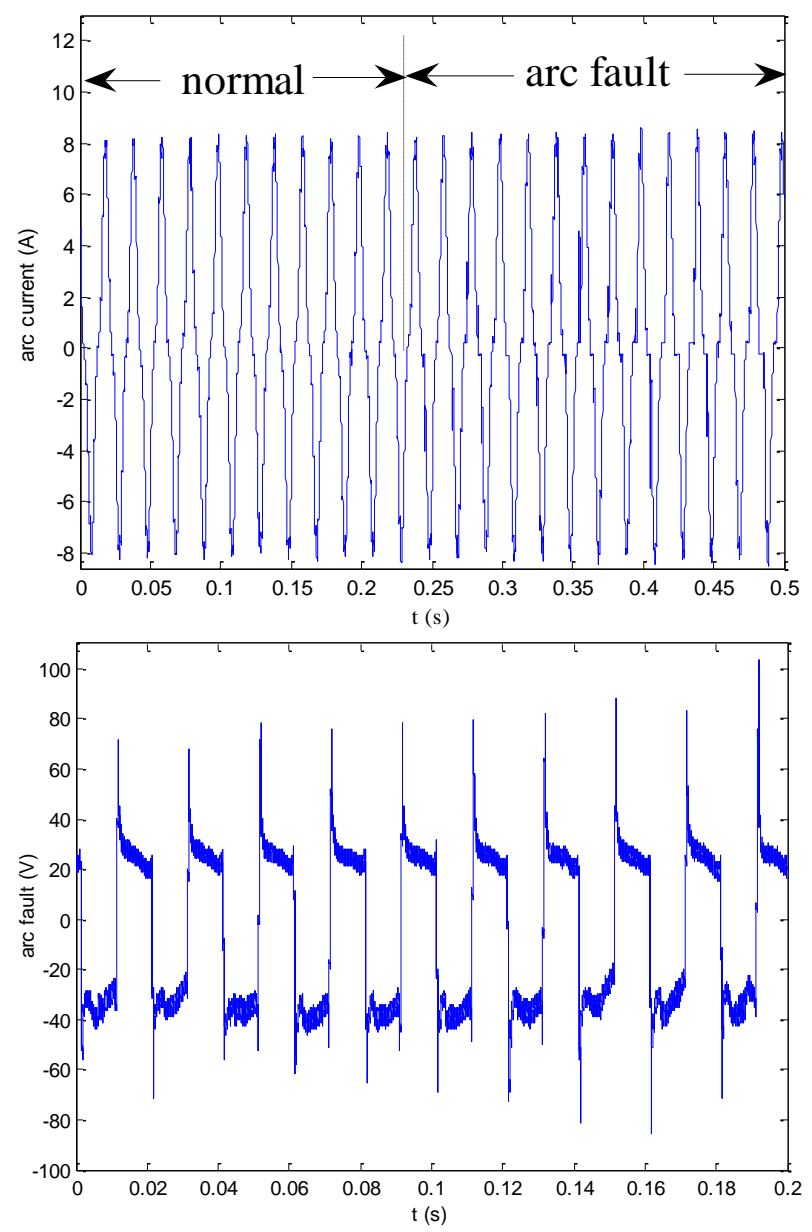

Figure 2. Arc fault current and voltage.
Experimental loads in those electric criterions consist of a $0.75 \mathrm{~kW}$ electrical hand drill, a $1.2 \mathrm{~kW}$ vacuum cleaner, a $2.2 \mathrm{~kW}$ air compressor, several $0.2 \mathrm{~kW}$ tungsten lamps, a $0.25 \mathrm{~kW}$ computer, several $0.2 \mathrm{~kW}$ dimming lamps, and so on. USB data acquisition system and Current transducers are used to sample a lot of load signals in circuits. The time-domain waveforms of series arc fault and normal mixed signals are shown in Fig. 2. Some series arc fault signals do not have obvious characteristics so that they cannot be easily identified from the strong surrounding interference through a stationary threshold. Consequently, it is hard to extract common characteristics from series arc faults of different loads.

\section{BLIND SOURCE SEPARATION THEORY}

Blind source separation is a novel theory in the field of fault signal analysis and processing, its main separation model is described in Fig. 3. And its mathematic model can be briefly set up as

$$
\boldsymbol{Y}(t)=\boldsymbol{M} \boldsymbol{X}(t)+\boldsymbol{D}(t) \text {. }
$$

Where $\boldsymbol{X}(t)=\left[x_{1}(t), x_{2}(t), \ldots, x_{\mathrm{L}}(t)\right]^{\mathrm{T}}$ is the vector of statistically independent signal variables which are named independent components, $\boldsymbol{M}$ is an unknown $\mathrm{K} \times \mathrm{L}$ matrix which is named mixed matrix, and $\boldsymbol{Y}(t)=\left[y_{1}(t)\right.$, $\left.y_{2}(t), \ldots, y_{\mathrm{K}}(t)\right]^{\mathrm{T}}$ is the vector of observed random variables, $\boldsymbol{D}(t)=\left[d_{1}(t), d_{2}(t), \ldots, d_{\mathrm{K}}(t)\right]^{\mathrm{T}}$ is the vector of interference. The observed random vector $\boldsymbol{Y}(t)$ is used to estimate both the mixed matrix $\boldsymbol{M}$ and the independent components $x_{i}(t)(i=1,2, \ldots, \mathrm{L})$. The output $\boldsymbol{Z}(t)$ is BSS separation result [10].

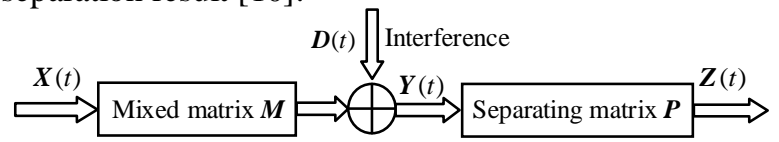

Figure 3. BSS separation model.

At first, the separating matrix $\boldsymbol{P}$ and the observation vector $\boldsymbol{Y}$ are considered as neural units. Under the constraint $E\left\{\left(\boldsymbol{P}^{\mathrm{T}} \boldsymbol{Y}\right)^{2}\right\}=\|\boldsymbol{P}\|^{2}=1$, the target of BSS algorithm is to obtain the extreme of $E\left\{F\left(\boldsymbol{P}^{\mathrm{T}} \boldsymbol{Y}\right)\right\}$, here $F$ is a given non square and nonlinear function. In the light of the Karush-Kuhn-Tucker conditions, the extreme of $E\left\{F\left(\boldsymbol{P}^{\mathrm{T}} \boldsymbol{Y}\right)\right\}$ are gotten at sites where

$$
E\left\{\boldsymbol{Y} f\left(\boldsymbol{P}^{\mathrm{T}} \boldsymbol{Y}\right)\right\}+\gamma \boldsymbol{P}=\mathbf{0} .
$$

Where $\gamma=E\left\{\boldsymbol{P}_{0}^{\mathrm{T}} \boldsymbol{Y} f\left(\boldsymbol{P}_{0}^{\mathrm{T}} \boldsymbol{Y}\right)\right\}$, the derivative of $F$ is $f$ and the value of $\boldsymbol{P}$ at the optimum is $\boldsymbol{P}_{0}$. According to Newton iterative method, equation (2) can be solved. The mark $U$ is used to denote the part on the left-hand side of (2), and $J\{U(\boldsymbol{P})\}$ is $U$ 's Jacobian matrix, then it can be gotten as [11]

$$
J\{\boldsymbol{U}(\boldsymbol{P})\}=E\left\{\boldsymbol{Y} \boldsymbol{Y}^{\mathrm{T}} f^{\prime}\left(\boldsymbol{P}^{\mathrm{T}} \boldsymbol{Y}\right)\right\}+\gamma \boldsymbol{I} .
$$

Where, $I$ is a unit matrix.

As $\boldsymbol{Y}$ is a whitened vector, the mean value of $\boldsymbol{Y} \boldsymbol{Y}^{\mathrm{T}} f^{\prime}\left(\boldsymbol{P}^{\mathrm{T}} \boldsymbol{Y}\right)$ in (3) can be calculated as

$$
\begin{aligned}
E\left\{\boldsymbol{Y} \boldsymbol{Y}^{\mathrm{T}} f^{\prime}\left(\boldsymbol{P}^{\mathrm{T}} \boldsymbol{Y}\right)\right\} & \approx E\left\{\boldsymbol{Y} \boldsymbol{Y}^{\mathrm{T}}\right\} \cdot E\left\{f^{\prime}\left(\boldsymbol{P}^{\mathrm{T}} \boldsymbol{Y}\right)\right\} . \\
& =E\left\{f^{\prime}\left(\boldsymbol{P}^{\mathrm{T}} \boldsymbol{Y}\right)\right\} \cdot \boldsymbol{I}
\end{aligned}
$$


According to Newton iterative method, the optimum result of (3) can be gotten, and the iterations are obtained as

$$
\left\{\begin{array}{l}
\boldsymbol{P}^{+}=E\left\{\boldsymbol{Y} f\left(\boldsymbol{P}^{\mathrm{T}} \boldsymbol{Y}\right)\right\}-E\left\{f^{\prime}\left(\boldsymbol{P}^{\mathrm{T}} \boldsymbol{Y}\right)\right\} \boldsymbol{P} \\
\boldsymbol{P}^{++}=\boldsymbol{P}^{+} /\left\|\boldsymbol{P}^{+}\right\|
\end{array} .\right.
$$

After iteration, researchers will obtain a new matrix $\boldsymbol{P}^{++}$. And the iteration will not stop until the whole algorithm become convergent. At last, the final matrix $\boldsymbol{P}^{++}$denotes BSS separation matrix $\boldsymbol{P}$. Therefore, $\boldsymbol{Z}=\boldsymbol{P}^{\mathrm{T}} \boldsymbol{Y}$. The main steps of BSS algorithm can be listed as follows:

1) The observation signal $\boldsymbol{Y}$ will be whitened firstly.

2) The estimate number of independent components is set as $\mathrm{Q}$, and $k=1$ is denoted as the sequence number of estimated component.

3) $\boldsymbol{P}_{k}$ is selected as a random initial weight vector.

4) Calculate $\boldsymbol{P}_{k}^{*}$ :

$$
\boldsymbol{P}_{k}^{*}=E\left\{\boldsymbol{Y} f\left(\boldsymbol{P}_{k}^{\mathrm{T}} \boldsymbol{Y}\right)\right\}-E\left\{f^{\prime}\left(\boldsymbol{P}_{k}^{\mathrm{T}} \boldsymbol{Y}\right)\right\} \boldsymbol{P}_{k} .
$$

5) Calculate $\boldsymbol{P}_{k}^{* *}$

$$
\boldsymbol{P}_{k}^{* *}=\boldsymbol{P}_{k}^{*}-\sum_{i=1}^{k-1}\left(\left(\boldsymbol{P}_{k}^{*}\right)^{\mathrm{T}} \boldsymbol{Y}_{i}\right) \boldsymbol{Y}_{i} \cdot
$$

6) Calculate $\boldsymbol{P}_{k}^{* * * *}$ :

$$
\boldsymbol{P}_{k}^{* * *}=\boldsymbol{P}_{k}^{* *} /\left\|\boldsymbol{P}_{k}^{* *}\right\| \cdot
$$

source 1

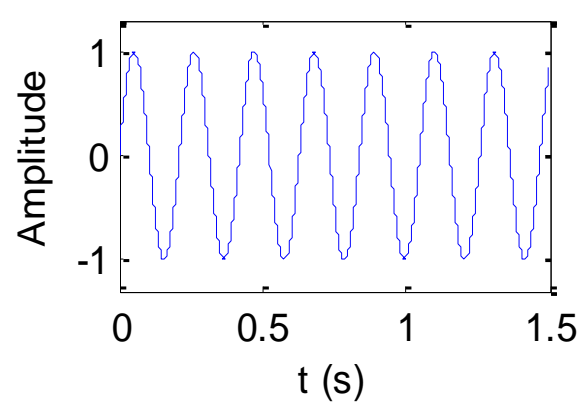

mixed signal 1

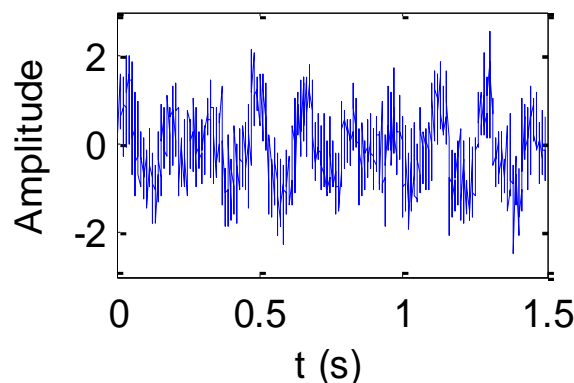

separated signal 1

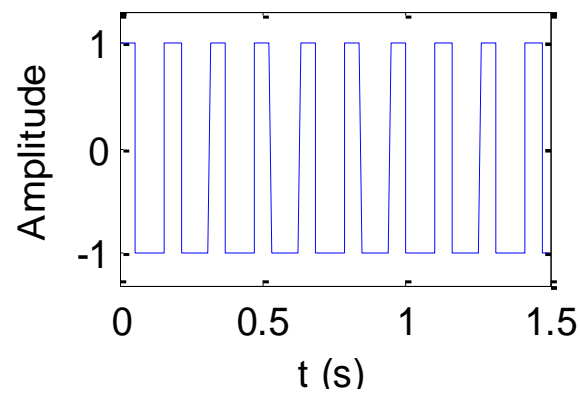

$\mathrm{t}(\mathrm{s})$
7) If $\boldsymbol{P}_{k}^{* * *}$ is not convergent, the algorithm will return back to the step 4 , and $\boldsymbol{P}_{k}^{* * * *}$ will be used to replace $\boldsymbol{P}_{k}$. Otherwise, it will output the estimated signals $\boldsymbol{y}_{k}=\left(\boldsymbol{P}_{k}^{* * * *}\right)^{\mathrm{T}} \boldsymbol{Y}$.

8) The sequence number $k=k+1$. If $k \leq \mathrm{Q}$, the calculation will return back to step 3 . Otherwise, the whole iteration algorithm will stop.

Three source signals, which contains sine wave, Gaussian random signal and rectangular wave, are used to prove the validity of the separation algorithm BSS. The sampling frequency of waves is $1 \mathrm{kHz}$. The mathematic expressions of that signals are listed as follows:

$$
\left\{\begin{array}{l}
s_{1}(t)=\sin (30 * t) \\
s_{2}(t)=\operatorname{randn}(1,1500) \\
s_{3}(t)=\operatorname{sgn}[\sin (40 * \mathrm{t}+0.14 \pi)-\sin (0.14 \pi)]
\end{array}\right.
$$

Where randn $(1,1500)$ returns an matrix containing pseudo-random values drawn from a normal distribution with mean zero and standard deviation one, 'sgn' is a sign function. They are shown in Fig. 4.

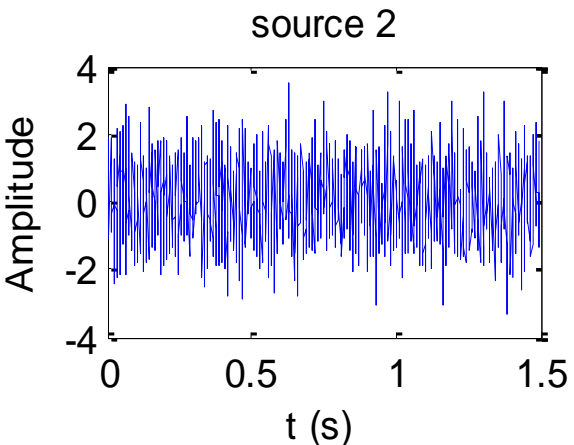

mixed signal 2

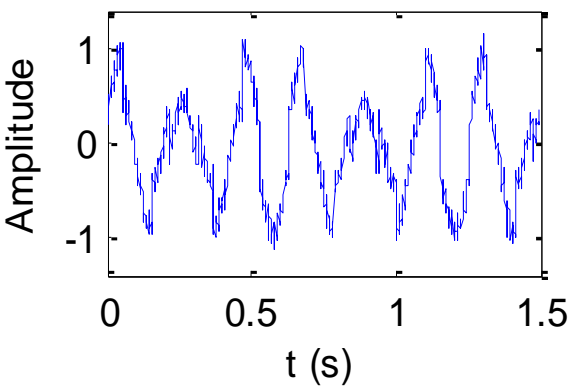

separated signal 2

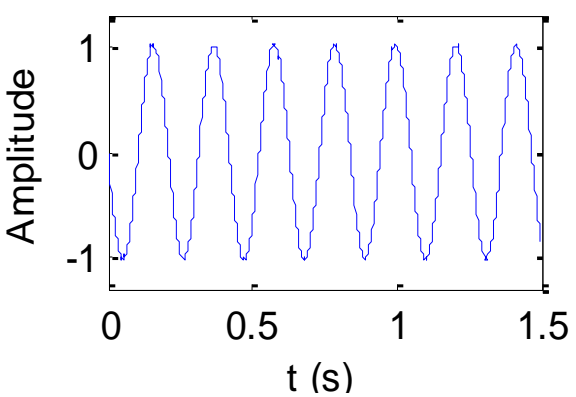

source 3

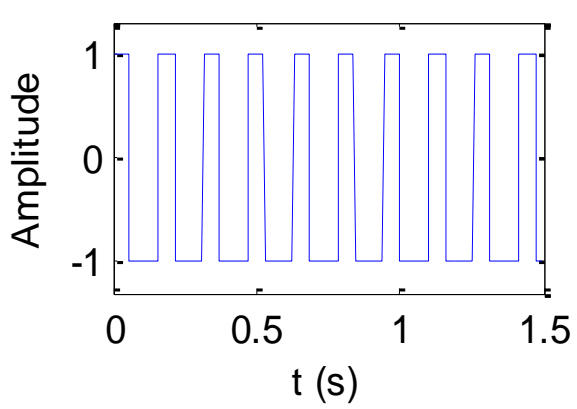

mixed signal 3

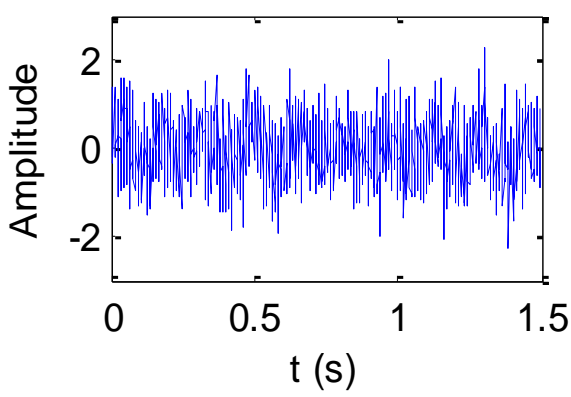

separated signal 3

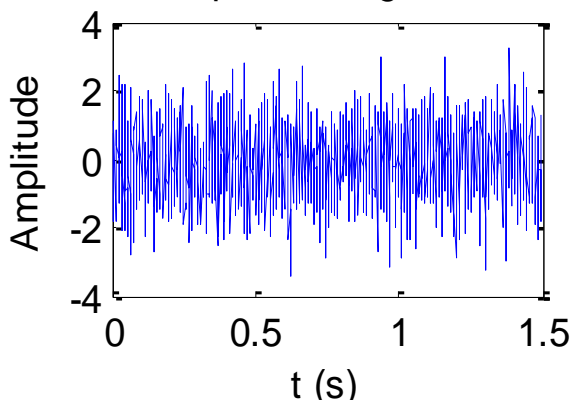

Figure 4. Simulation signals of BSS. 
During the verification of BSS, a random mixed matrix $\boldsymbol{M}$ is generated, and it is listed as follows:

$$
\boldsymbol{M}=\left[\begin{array}{lll}
4.10705 & -2.26021 & -3.00223 \\
1.88925 & -3.17421 & -1.10146 \\
1.45384 & -5.21179 & -2.78058
\end{array}\right] .
$$

The three sources are firstly mixed by mixed matrix $\boldsymbol{M}$. Then, three mixed signals are generated which are shown in Fig. 4. As the mixed matrix $\boldsymbol{M}$ is a random matrix, mixed signals have very strong randomness; they are different in each simulation. Actually, they are treated as observed mixed signals to verify the validity of BSS. Although the observed signals are different from last simulation, they can also be separated. The separation results are shown in Fig. 4. Due to the three sources are independent components in statistics, they can also be successfully separated from mixed signals without some waveform distortion. Consequently, BSS theory may be applied to separate series arc fault mixed signals.

\section{SERIES ARC FAULT CHARACTERISTICS}

\section{A. The separation of series arc fault mixed signals}

During the analysis of series arc faults, researchers should carefully pay attention to diversity, randomness and concealment of fault currents. There are many kinds of loads in low voltage circuits, their current features are not the same, particularly in series arc fault states. The generation site, time and intensity of series arc faults have some randomness. The accuracy of data acquisition and the reliability of data are usually impacted by load states, strong background interference and some complex external working environment. There are also some frequency aliasing between different signals. Some useful characteristics may be submerged in load currents which are acquired as mixed signals. It is hard to discriminate from them just use frequency filtration. Therefore, BSS theory is introduced to separate series arc fault mixed currents.
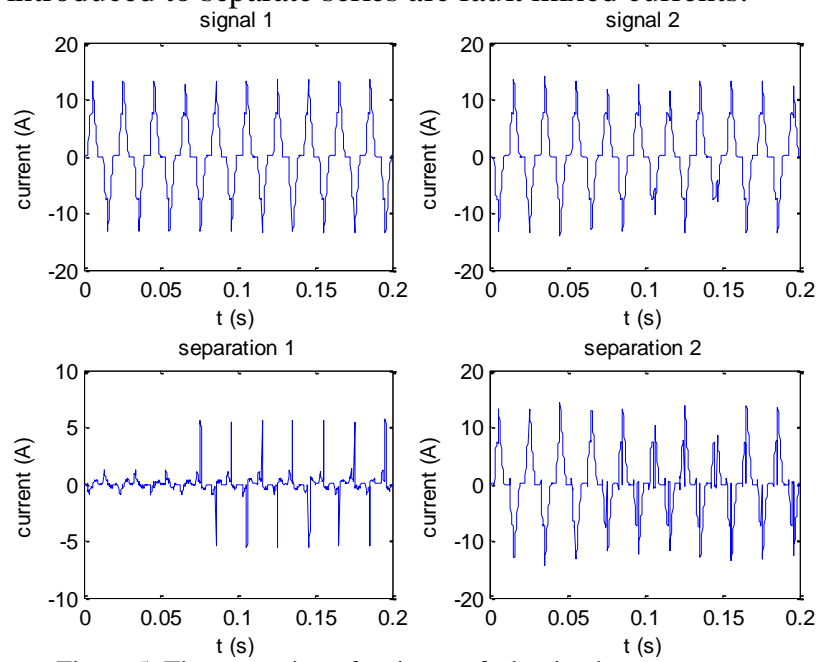

Figure 5. The separation of series arc fault mixed currents.

Currents of dimming lamps are selected to verify BSS as shown in Fig. 5. The separation components of series arc fault mixed currents are also shown in Fig. 5. The distortion components and the effect of BSS methodology are very obvious. The useful components of series arc faults can be quickly separated to well avoid other components' aliasing and restrain interference. And separation components can be used for further analysis to accurately identify series arc faults from load states.

\section{B. Harmonic analysis}

To quantitatively describe the separation component characteristics of series arc faults, harmonic analysis is introduced to study the series arc fault characteristics. Harmonic analysis is a frequency domain analysis method, and different harmonic amplitudes and phases can be separated from original signals through this method. Separated signal is expanded to discrete Fourier series through mathematical expression:

$$
X\left(\alpha w_{0}\right)=\frac{1}{C} \sum_{n=0}^{C-1} x(n) * e^{-j * *^{*} \frac{2 \pi}{C} *_{n}} \quad \alpha=0,1,2, \ldots, C-1 .
$$

Where $w_{0}$ is the angular frequency, $X\left(\alpha w_{0}\right)$ is the $\alpha$-th harmonic component, $x(n)$ is the separated current, $C$ is the total number of sampling.

When series arc fault occurs in vacuum cleaner circuit, current will distort, and its harmonic will also change a lot. Fault current spectrum is shown in Fig. 6 . From analysis of different load harmonics, it is not difficult to find that odd harmonics are bigger than even ones. The 3rd, 5th, 7th, 9th and 11th harmonic component is always relatively big. Fault arcs are similarly like nonlinear loads. When nonlinear loads are connected into circuits, impedance characteristics of current will change, harmonics will change obviously. Similarly, fault arcs can also promote the addition of current harmonics.

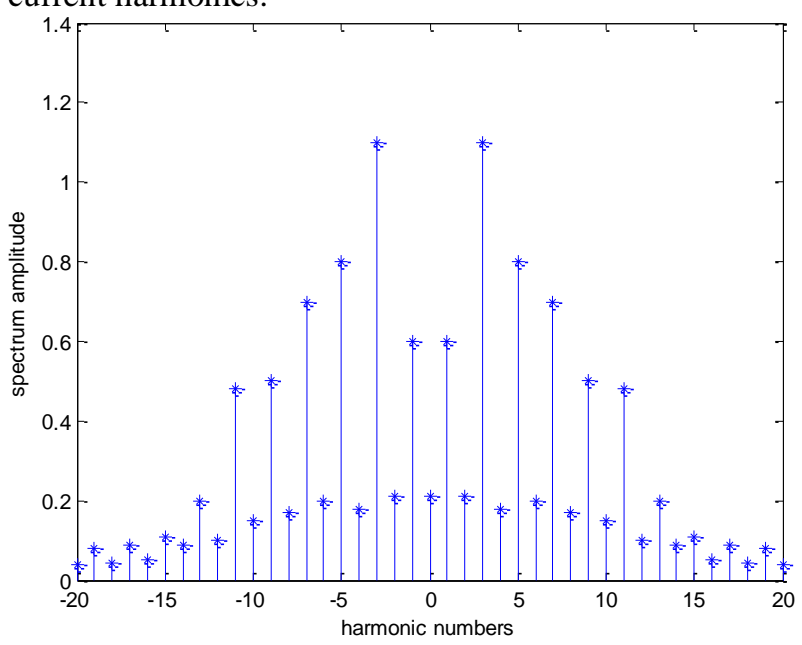

Figure 6. Current spectrum of vacuum cleaner arc fault.

Harmonic is usually described by harmonic ratio $(H R)$. According to $H R$ 's definition, $H R$ is a percent. It can be defined as: In periodic AC circuits, the root mean square of first harmonic component is divided by the root mean square of $\alpha$-order harmonic component. Mathematic expression of current harmonic ratio can be described as: 


$$
H R I_{\alpha}=\frac{I_{\alpha}}{I_{1}} \times 100 \% .
$$

According to the analysis results of harmonic component, it is easy to conclude that odd $H R$ will also increase a lot when series arc fault occurs in circuit. In order to count all odd $H R$, the $H R$ sum of 3rd, 5th, 7th, 9th and 11th harmonic component is supposed to calculate. Its mathematic expression can be described as:

$$
H R_{s o}=\frac{I_{3}+I_{5}+I_{7}+I_{9}+I_{11}}{I_{1}} \times 100 \% .
$$

As $H R_{s o}$ is the accumulation of odd $H R$, its amplitude change will be more obvious. A large number of series arc faults occurred in different kinds of load circuits are analyzed to research the common characteristics. The tungsten lamp current's $H R_{s o}$ is shown in Fig. 7. When tungsten lamp works in normal condition, current $H R_{s o}$ is small, $H R_{s o}$ is almost under $10 \%$, and it keeps stability. However, when series arc faults occur in circuits, $H R_{s o}$ becomes instability and random fluctuation, it changes rapidly and randomly. Amplitude of $H R_{s o}$ increases in multiples.

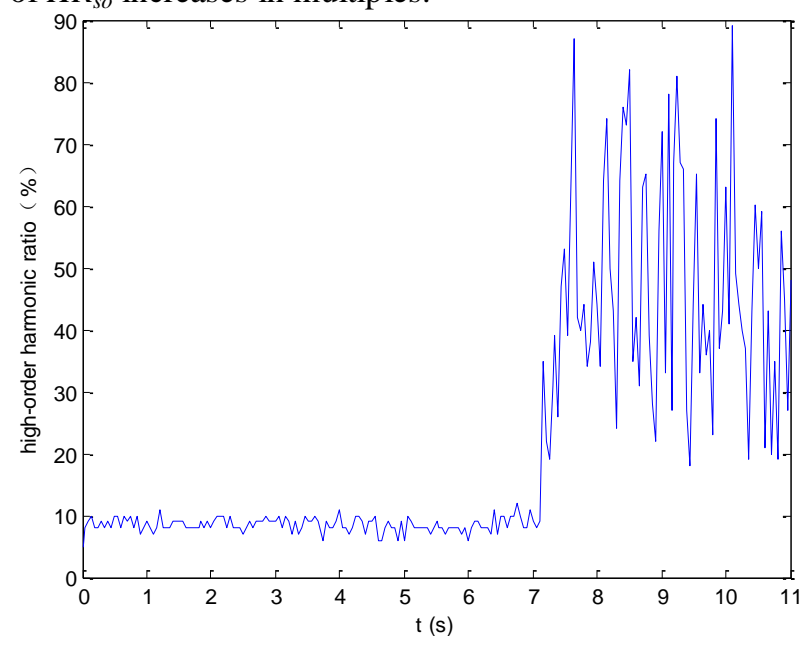

Figure 7. Odd order $H R_{s o}$ analysis of tungsten lamp current.

Duing the using of an electrical hand drill, dimming lamps, etc., the working states are usually regulated, such as speed regulation, currents will also change. However, high order $H R$ does not change disorder, that changes keep stability. The vacuum cleaner current's $H R_{s o}$ is shown in Fig. 8.

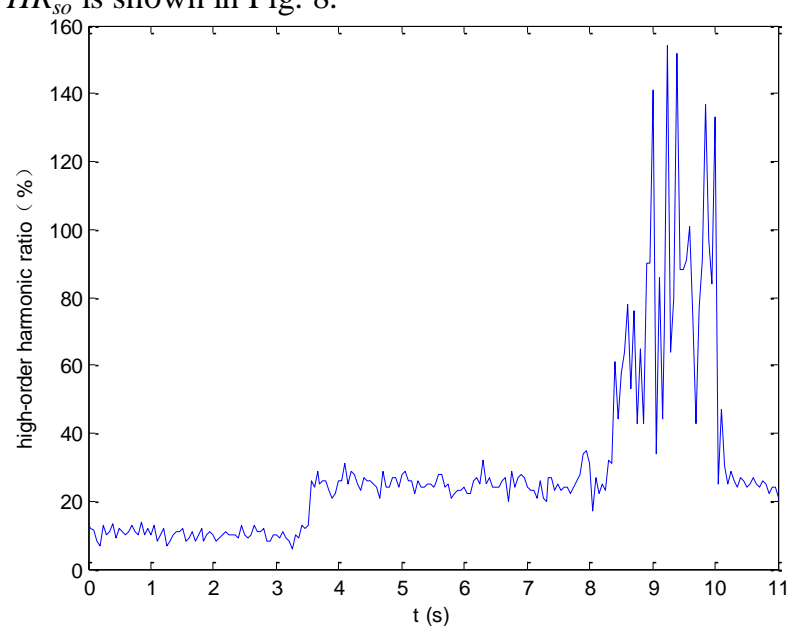

Figure 8. Odd order $H R_{s o}$ analysis of vacuum cleaner current.
When the vacuum cleaner reduces the speed, harmonics increase a little, and amplitude of $H R_{s o}$ also increases a little but keeps stability. Nevertheless, when series arc faults occur in vacuum cleaner circuit, $H R_{s o}$ fluctuate tempestuously without any regular.

In order to analyze harmonic characteristics of series arc faults comprehensively, several loads consist of a vacuum cleaner, tungsten lamps and dimming lamps are selected to work in parallel. Relative to current $H R$ of single load, current $H R$ of parallel loads is bigger although the loads work in normal condition. The several parallel load currents' $H R_{s o}$ is shown in Fig. 9. As a result of nonlinear loads, the currents' total $H R_{s o}$ increases a little. However, its change is steady-going. $H R_{s o}$ keeps in a small certain range which is quite different from series arc faults $H R_{s o}$. When series arc faults occur in parallel load circuits, $H R_{s o}$ changes rapidly and randomly. Sometimes the amplitude increases to $182 \%$, and the mean value of total $H R_{s o}$ is more than $79 \%$. In a word, harmonic characteristics are quite obvious. They can be used to clearly identify series arc faults from load states. Consequently, it is very significant for arc fault detection. It also provides favorable bases for arc fault detection.

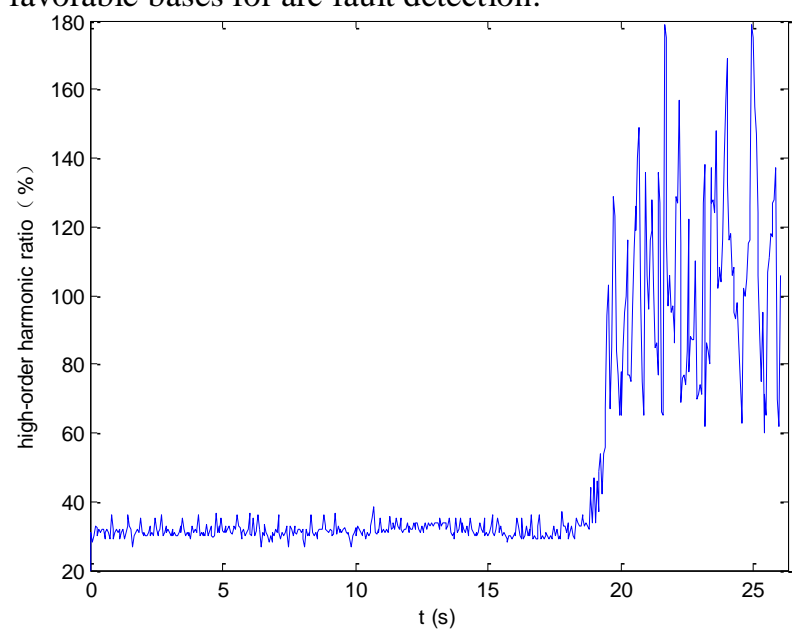

Figure 9. Odd order $H R_{s o}$ analysis of several parallel load currents.

\section{CONCLUSIONS}

In order to detect series arc faults of different loads in circuits, it is very important to research about common characteristics of series arc faults. In this paper, a low voltage experimental platform is firstly set up for systematic study of arc faults. The main conclusions are listed as follows:

1) When series arc faults occur in low voltage circuits, currents will distort a little. The degree of current distortions is different among different load circuits.

2) Arc fault currents contain distortions can be successfully separated from the observed mixed signals through BSS. They are used to research common characteristics of series arc faults.

3) Compared with normal load currents, harmonics of arc fault currents changes a lot. The amplitudes of odd harmonic components increase rapidly, and $H R_{s o}$ fluctuate tempestuously with inconstancy. Based on 
those characteristics of current harmonics, series arc faults in different load circuits can be well discriminate from load states.

\section{ACKNOWLEDGEMENTS}

This work was supported by the Fujian Natural Science Foundation (2012JH01214), the Fujian Industry-University Cooperation Science \& Technology Major Project (2012H6013), the Xiamen Science \& technology project of China (Z1424045) and the Quanzhou Science \& technology project of China (2014Z114).

\section{REFERENCES}

[1] G. Si, "Analysis on the national electrical fire situation and characteristics of the past five years," Fire Science and Technology, vol. 33(5), 2014, pp. 570-52.

[2] Fire Department of Ministry of Public Security, China Fire Services 2014, Yunnan People's Publishing House: Kunming, 2014, pp. 396-407.

[3] D. G. George, W. Kon and D. Robert, "More about ArcFault Circuit Interrupters," IEEE Transaction on Industry Applications, vol. 2, 2004, pp. 1306-1313.

[4] K. Phipps, T. Cooke, D. Dorr and P. Keebler, "Method to Design Arc Fault Detection Algorithm Using FPGA," Proc. 2011 IEEE 57th Holm Conference on Electrical Contacts, IEEE Press, Sep. 2011, pp. 1-5.
[5] J. H. Yang, R. C. Zhang and H. Y. Fang, "Early detecting of fault arcs using Lyapunov exponents," Advanced Technology of Electrical Engineering and Energy, vol. 27(2), 2008, pp. 56-58.

[6] J. K. Charles, "Electromagnetic Radiation Behavior of Low-Voltage Arcing Fault," IEEE Transactions on Power Delivery, vol. 24, 2009, pp. 416-423.

[7] P. Giuseppe, M. Luigi and L. Marco, "Simplified ArcFault Model: The Reduction Factor of the Arc Current," IEEE Transactions on Industry Applications, vol. 49, 2013, pp. 1703-1710.

[8] J. Lezama, P. Schweitzer, S. Weber, E. Tisserand and P. Joyeux, "Modeling of a domestic electrical installation to arc fault detection", Proc. 58th IEEE Holm Conference on Electrical Contacts, IEEE Press, Sep. 2012, pp. 1-7.

[9] H. R. Wu, X. H. Li, D. Stade and H. Schau, "Arc fault model for low voltage AC systems," IEEE Transactions on Power delivery, vol. 20, 2005, pp. 1204-1205.

[10] A. Hyvarinen, "Fast and robust fixed-point algorithms for independent component analysis," IEEE Transactions on Neural Networks, vol. 10(3), 1999, pp. 626-634.

[11]Z. Y. Wang, C. Jin, G. M. Dong and Z. Yu, "Constrained independent component analysis and its application to machine fault diagnosis," Mechanical Systems and Signal Processing, vol. 25(7), 2011, pp. 2501-2512. 